Equilibrium Res Suppl. 3, 1972

\title{
HISTOCHEMICAL DEMONSTRATION OF VESTIBULAR EFFERENT FIBERS
}

\author{
Tetsuo IsHII
}

\section{INTRODUCTION}

In the field of light microscopy, two techniques are used for the demonstration of the course of the efferent nervous system in the peripheral vestibular sense organs. One method is staining the degenerated nerve fibers using the Nauta silver technique (Gacek ${ }^{3}$, 1966), after sectioning the efferent bundles centrally. The other is the histochemical demonstration of $\mathrm{AChE}$ in the nerve fibers. The efferent nerve fibers in the inner ear are known to present strong AChE activity, as Churchill et al. ${ }^{1}$, (1956) found AChE activity in the efferent bundles of the cochlea. Histochemical demonstration of AChE activity is helpful in pursuing the morphological course of the efferent innervation in the inner ear, though AChE-positive fibers, which apparently do not belong to Rasmussen's olivo-cochlear bundle, penetrate the inner ear directly through the glial-Schwann sheath junction (Ishii et al. $\left.{ }^{8,9}, 1967 \mathrm{a}, 1967 \mathrm{~b}\right)$. The author has previously reported AChE activity in the human and monkey inner ears, utilizing techniques to demonstrate AChE activity in the vestibular organs. In the present study, distribution of AChE activity in the vestibular system, with stress on the course of the efferent fibers, is reported.

\section{MATERIALS AND METHODS}

Five cats and three squirrel monkeys were used. Animals were injected with sodium pentobarbital, thoracotomized and perfused through the aorta with ice-cold physiological saline solution for $10 \mathrm{~min}$. They were then perfused with cold $4 \%$ formol-calcium solution at $\mathrm{pH}$ 7.1. The temporal bones were removed and decalcified with cold $10 \%$ EDTA (ethylenediamine tetraacetate) solution buffered with phosphate at $\mathrm{pH} 7.4$ for 5 to 14 days. After decalcification, the temporal bones were sectioned in a cryostat at $12 \mu$. The sections were thawed on the cover-slips and dried at room temperature, and then were incubated in the substrates prepared after Karnovsky \& Roots ${ }^{5}$, (1964) and Gomori ${ }^{4}$, (1952). After incubation for 2 hours, the sections were briefly rinsed in distilled water and mounted on microscopic slides with PVP (Polivinyl Pyrrolidone) solution or Canada Balsam after dehydration with graded alcohol. The inhibitory test to specify cholinesterase activity (Pepler \& Pearse $\left.{ }^{11}, 1957\right)$, was performed; eserine salicylate solution (in a final concentration, $\left.10^{-4} \mathrm{M}\right)$ as an inhibitor for $\mathrm{AChE}$ and $\mathrm{ChE}$ was used. Silver nitrate $\left(10^{-3}\right.$ to $10^{-6} \mathrm{M}$ ) and iso-OMPA (tetra-isopropylpyrophosphoramide, $10^{-3}$ to $10^{-6} \mathrm{M}$ ) were used before and after incubation for the inhibition of ChE.

From the Department of Otolaryngology, Teikyo University, School of Medicine, Tokyo 


\section{RESULTS}

AChE activity was demonstrated as distinct brown precipitates. Histochemical re-

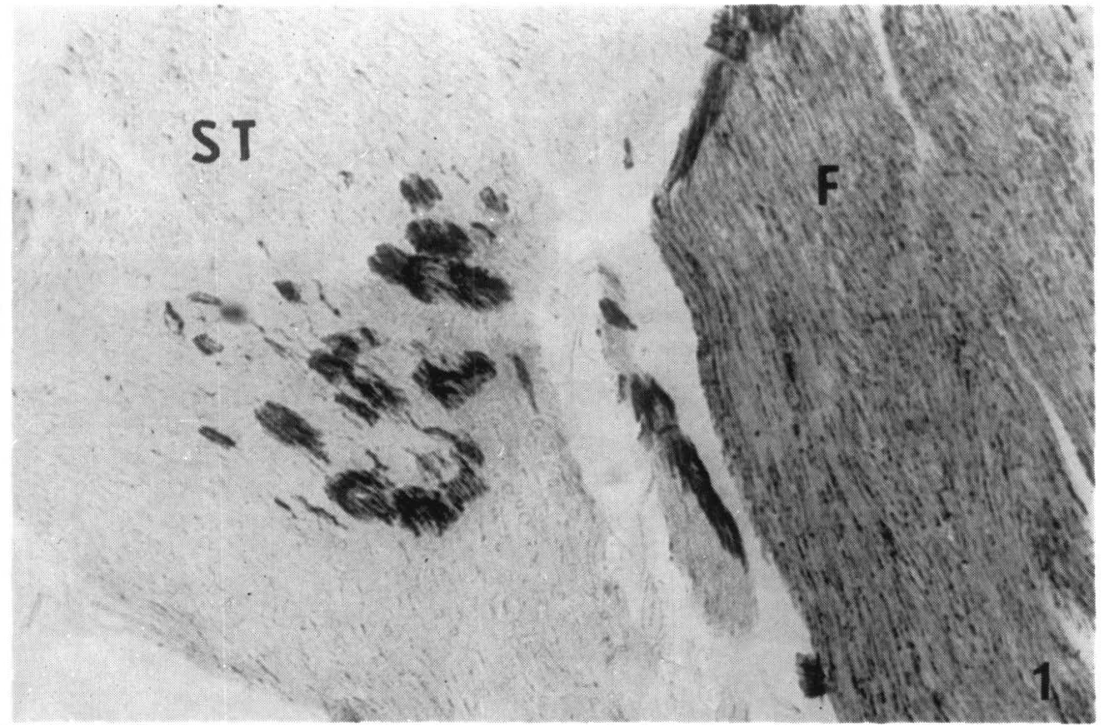

Fig. 1. Cross section of statoacoustic (ST) and facial (F) nerves. AChE-active nerves are observed to consist of several bundles in the statoacoustic nerve. $\times 100$.

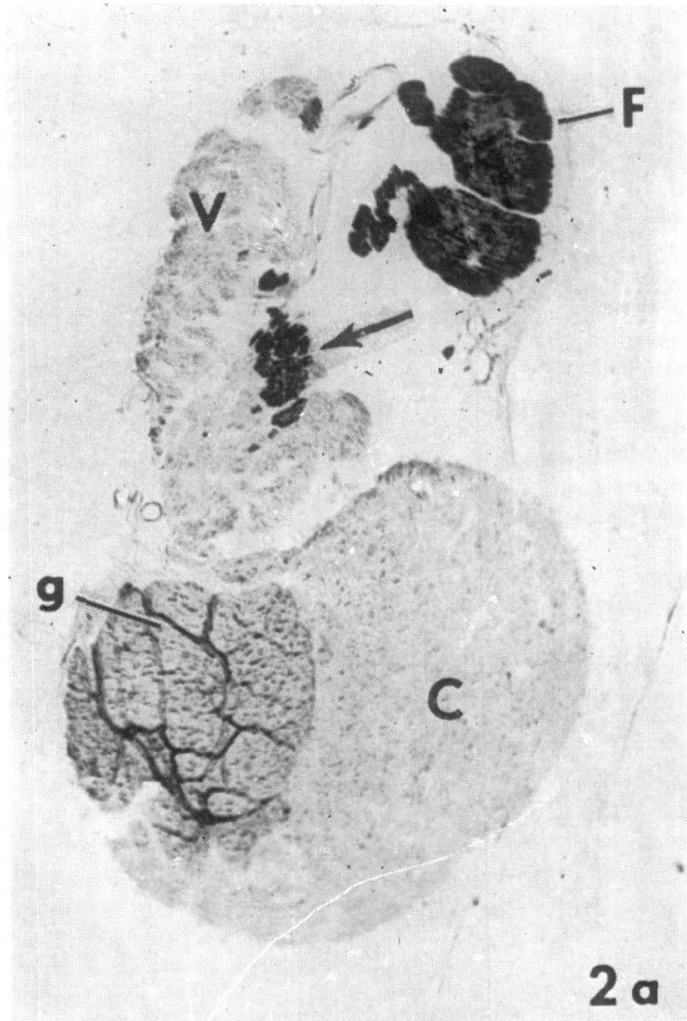

Fig. 2a. Cross section of the internal auditory meatus. Arrow points to efferent bundle with high AChE activity. F : facial nerve. $V$ : vestibular nerve. $\mathrm{C}$ : cochlear nerve. $\mathrm{g}$ : glial-Schwann sheath junction. $\times 38$. 
action for AChE and ChE was inhibited by eserine salicylate. Silver nitrate $\left(10^{-5} \mathrm{M}\right)$ and iso-OMPA $\left(10^{-3} \mathrm{M}\right)$ failed to inhibit the histochemical reaction in the efferent fibers. These findings indicate that the enzyme activity demonstrated was exclusively AChE.

AChE activity in the statoacoustic nerve, central to the glial Schwann-sheath junction, was found located in several nerve bundles which showed a scattered distribution (Fig. 1). Peripheral to the glial-Schwann sheath junction, these AChE-active bundles united in one bundle in the vestibular nerve, size $160 \times 320 \mu$ (Fig. 2a). The AChE-positive nerve started to break into small fibers at the point where the vestibular nerve divides into the superior and inferior divisions (Fig. 2b). The course and size of these AChEpositive nerve fibers in the vestibular nerve are apparently similar to those of Rasmussen's olivocochlear bundle, though more than half of these bundles distribute in the vestibular organs. Scattered distribution of AChE-positive nerve bundles was seen in the ampullar, saccular and utricular nerves. Besides the vestibular efferent fibers, AChE-active fibers were sparsely discerned in the vestibular nerve. In the cat, these AChE-positive fibers

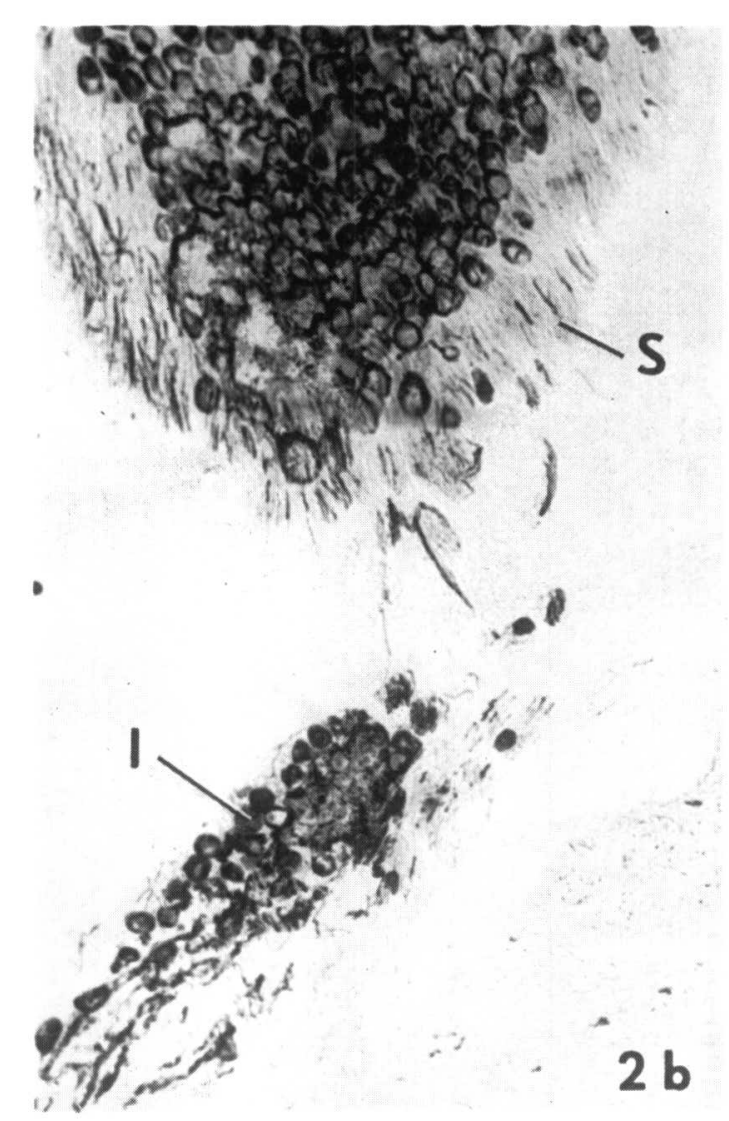

Fig. 2b. Vestibular nerve divides into superior (S) and inferior (I) portions. Vestibular ganglion cells are seen to show intense AChE, though they are of afferent nature. AChE-positive efferent fibers scatter throughout the nerve. $\times 75$. 
were very sparse, but apparently they are independent of the already known efferent fiber tracts. Fig. 3 illustrates the AChE-positive fibers distributed sparsely throughout the vestibular nerve.

AChE-positive fibers were detected as far peripherally as the sensory epithelia (Fig. $4 a, b, c)$ and they branch in their course and seem to terminate as nerve ending-like round structures which are located under the sensory epithelia. In the saccular nerve, AChEpositive fibers seemed quantitatively the same as in Voit's bundle coming from the superior

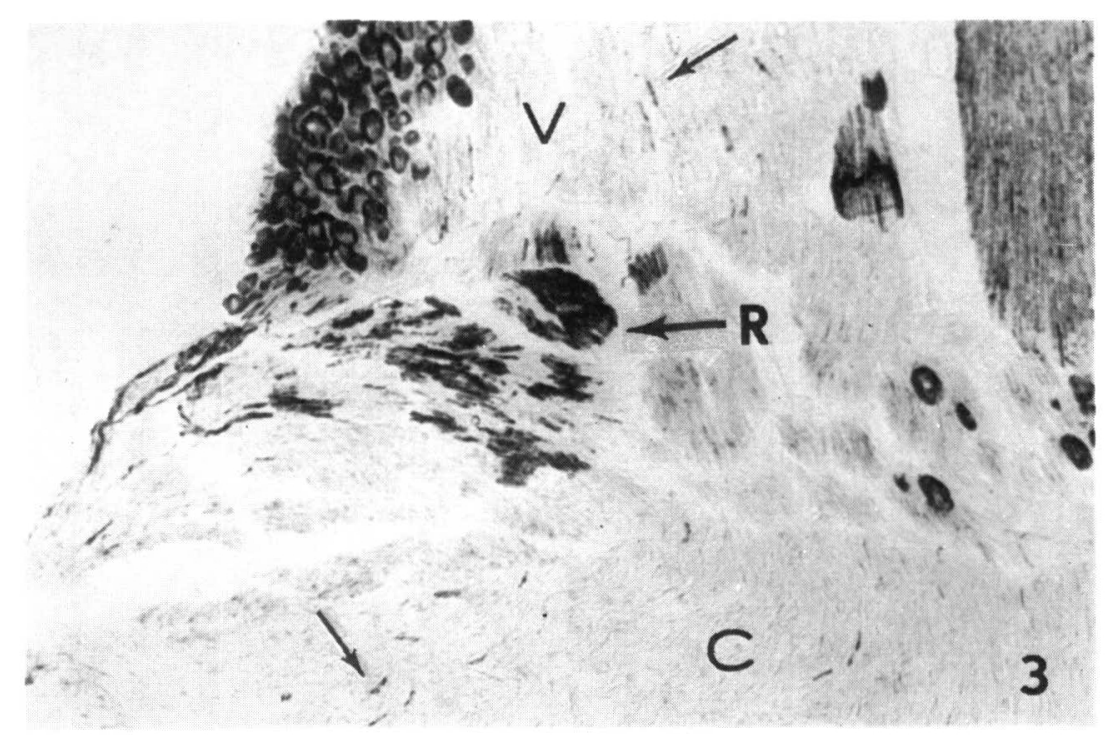

Fig. 3. Cross section of statoacoustic nerve. AchE-active nerve fibers (arrows) distributed in the vestibular ( $V$ ) and cochlear (C) nerves. They are apparently independent of Rasmussen's efferent bundle (R) $\times 90$.

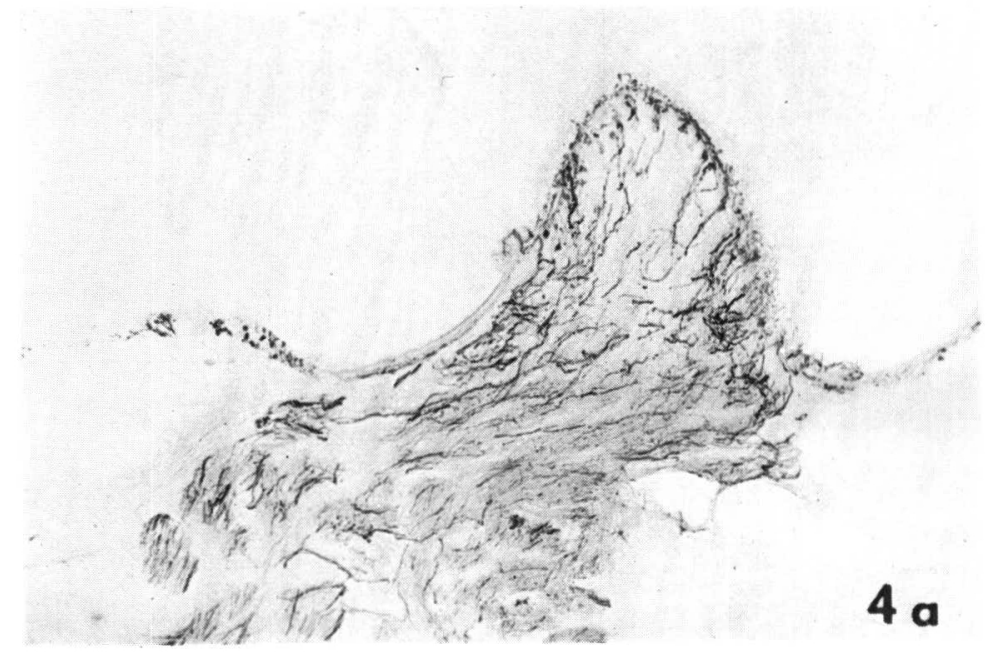

Fig. 4a. AChE-active fibers are found to distribute sparsely throughout the nerve and nerve endings of the lateral crista ampullaris. $\times 100$. 
portion of the vestibular nerve.

\section{DISCUSSION}

Since Churchill ${ }^{1}$ et al., (1956) first demonstrated intense AChE activity in the olivocochlear bundle, many histochemical studies have been carried out to detect the course of efferent nerve fibers in the cochlea. AChE activity in the vestibular efferent fibers has also been demonstrated (Dohlman et al. ${ }^{2}$, 1958; Ireland \& Farkashidy $^{6}$, 1961; Rossi ${ }^{12}$, 1961; Nomura et al. ${ }^{10}$, 1965). The author ${ }^{8}$ (1967) demonstrated AChE activity in the efferent

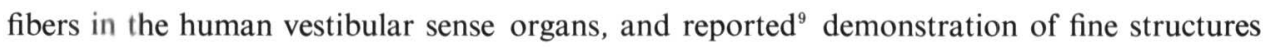
in the vestibular efferent fibers using vital perfusion-prefixation with $4 \%$ formol-calcium

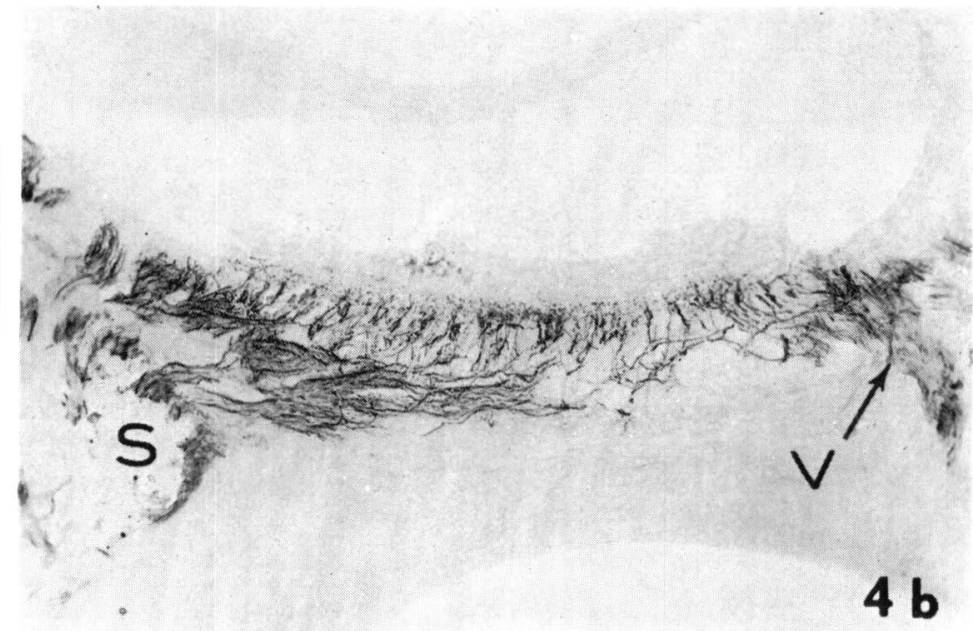

Fig. 4b. AChE activity in the saccule.

$\mathrm{S}$; saccular nerve. $\mathrm{V}$; Voit's bundle. $\times 80$.

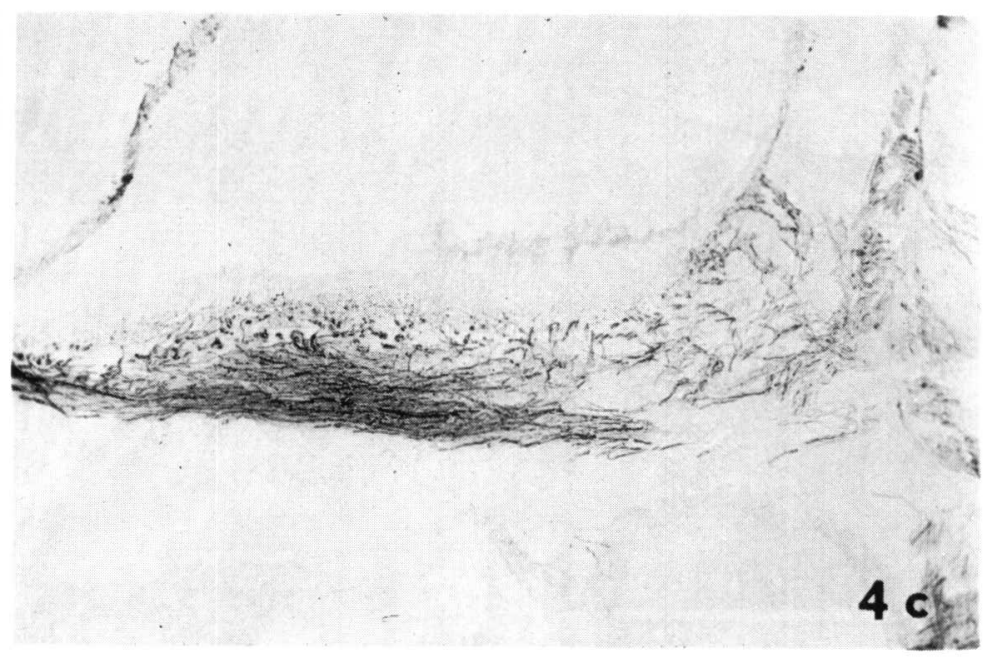

Fig. 4c. AChE-positive fibers distribute in the nerve endings in the utricle. $\times 80$ 
solution and decalcification with EDTA. The removal of erythrocytes was preferable, because they are known to show intense AChE activity. Prefixation is also essential for demonstrating the delicate structures of $\mathrm{AChE}$-active fibers, otherwise diffusion of the enzyme and/or histochemical deposits may occur. Prefixation of the inner ear also helps to prevent diffusion during the long period of decalcification with EDTA solution. Decalcification with EDTA at $4^{\circ} \mathrm{C}$ minimized distortion of the membraneous labyrinth, when the specimens were sectioned in a cryostat.

The course of efferent fibers is a group of several bundles at the root of statoacoustic nerve, then these bundles converge to form one large bundle. As described elsewhere (Gacek $\left.{ }^{3}, 1966\right)$, the vestibular efferent fibers begin to scatter where the vestibular nerve divides into the superior and inferior divisions. These efferent fibers end beneath the neuroepithelium of the cristae ampullares and otolith organs. Definite conclusion has not yet been drawn concerning the role of these vestibular efferent fibers, but the significance of the olivo-cochlear bundle, based on various assumptions, has been discussed.

It is interesting to note that sparse AChE-active fibers are seen in the vestibular nerve, which apparently does not belong to Rasmussen's bundle. It was reported that these fibers were seen in the cochlear nerve in man (Ishii et al $.^{8}, 1967$ ) and monkey (Ishii et al. $\left.{ }^{9}, 1967\right)$, and were confirmed to be of efferent nature by sectioning the cochlear nerve centrally. Though the experiment of sectioning the vestibular nerve has not been performed, the AChE-active fibers in the vestibular nerve were presumably efferent. The origin of these fibers must be detected in the future.

Another possible source of efferent components in the vestibule is vasomotor fibers. AChE-positive vasomotor fibers were reported to innervate the labyrinthine and common cochlear arteries, while the anterior vestibular artery failed to present AChE-active periarterial nerves (Ishii ${ }^{7}$, 1971). The vessels in the cristae and otolith organs show no AChEpositive perivascular nerves. Adrenergic fibers around the vessels in the vestibule have not yet been reported, while Spoendlin \& Lichtensteiger ${ }^{13}$ (1966) described the presence of adrenergic fibers in the vestibular nerve independent of the perivascular nerves.

The cat efferent bundle is a group of bundles, estimated $160 \times 320 \mu$ in the cat vestibular nerve, while the olivo-cochlear bundle is a nerve with a diameter of $50 \mu$ (unpublished data). Considering these findings, the vestibular sense organs are innervated by many more efferent fibers than the cochlea. Further work is needed to elucidate the functional significance of these vestibular efferent fibers.

\section{SUMMARY}

The histochemical demonstration of acetylcholinesterase (AChE) activity was observed to be well localized in the efferent nerve fibers in the inner ear. The distribution of $\mathrm{AChE}$ activity in the nerve fibers of cat and squirrel monkey were reported. The vestibular efferent fibers showed a similar course to that reported elsewhere, although AChEactive fibers were sparsely distributed in the vestibular nerve. These fibers did not apparently belong to the well-known efferent nerve fibers in the inner ear. The author em-

Equilibrium Res Suppl. 3 
phasized the necessity of the vital perfusion-prefixation and decalcification of the experimental animals, in order to obtain good preservation of the specimens and well-localized AChE activity.

\section{REFERENCES}

1) Churchill, J. A., Schuknecht, H. F. \& Doran, R.: Acetylcholinesterase activity in the cochlea. Laryngoscope 66: 1-15, 1956.

2) Dohlman, G. F., Farkashidy, J. \& Salonna, F.: Centrifugal nerve-fibers to the sensory eqithelium of the vestibular labyrinth. J. Laryng. 72:984-991, 1958.

3) Gacek, R. R.: The vestibular efferent pathway (Ed. Wolfson, R. J. The vestibular system and its diseases) Univ. Penn. Press. 99-116, 1966.

4) Gomori, G.: Microscopic histochemistry, principle and practice. Univ. Chicago Press. 211, 1952.

5) KARnovsky, M. J. \& RooTs, L.: A "direct-coloring" thiocholine method for cholinesterases. J. Histochem. Cytochem. 12: 219-220, 1964.

6) Ireland, P. E. \& Farkashidy, J.: Studies on the efferent innervation of the vestibular endorgans. Trans. Amer. Otol. Soc. 49: 20-30, 1961.

7) IsHII, T.: Acetylcholinesterase activity in the perivascular nerve plexus of the basilar and labyrinthine arteries. Acta Otolaryng. (Stockh.), 72: 281-287, 1971.

8) Ishit, T., Murakami, Y. \& Balogh, K. Jr.: Acetylcholinesterase activity in the efferent nerve fibers of the human inner ear. Ann. Otol. (St. Louis), 76: 69-83, 1967.

9) Ishit, T., Murakami, Y. \& GaCEK, R. R.: Histochemical study of the acetylcholinesterase activity in the inner ear of the squirrel monkey. Acta Otolaryng. (Stockh.), 64: 267-279, 1967.

10) Nomura, Y., GaceK, R. R. \& Balogh, K. Jr.: Efferent innervation of vestibular labyrinth. Arch. Otolaryng. (Chicago), 81:335-339, 1965.

11) Pepler, W. J. \& Pearse, A. G. E.: The histochemistry of the esterases of the hypothalamic nuclei. J. Neurochem. 84: 141-219, 1946.

12) Rossi, G.: L'acetylcholinesterase au cours du developpement de l'oreille interne du cobaye. Acta Otolaryng. (Stockh.), Suppl. 170, 1961.

13) Spoendlin, H. \& Lichtensteiger, W.: The adrenergic innervation of the labyrinth. Acta Otolaryng. (Stockh.), 61: 423-434, 1966.

(Received April 3, 1972) 